\title{
NOTA SOBRE EL TIPO, LA IDENTIFICACION Y LA LOCALIDAD DEL TIPO DE QUERCUS OBLONGIFOLIA VAR. PALLIDINERVIS (FAGACEAE) EN CHIHUAHUA
}

\author{
RichaRd SPELLENBERG \\ Dept. of Biology, New Mexico State University \\ Las Cruces, NM 88003-0001
}

\begin{abstract}
RESUMEN
Cuando Trelease describió la variedad Quercus oblongifolia Torr. var. pallidinervis Trel. indicó que el tipo se habia fotografiado en el herbario de "Washington", pero la muestra que ilustró se descubrió recientemente en el Herbario Gray. Por consiguiente esta muestra representa el holotipo; un ejemplar diferente de la misma colección se encuentra en el herbario US y corresponde al isotipo. Tomando en cuenta colecciones recientes y la literatura sobre el tema se determina aquí a esta variedad como sinónimo de Quercus depressipes Trel. y la localidad del tipo es la Sierra de la Breña en el noroeste de Chihuahua.
\end{abstract}

\section{ABSTRACT}

When Trelease described the variety Quercus oblongifolia Torr. var. pallidinervis Trel. he indicated that the type was photographed in "Washington", but the specimen that he illustrated was recently discovered to be in the Gray Herbarium. It is considered to be the holotype; a different specimen from the same collection resides in the US National Herbarium and is considered to be an isotype. Based on recent collections and the literature, the variety is determined to be a synonym of Quercus depressipes Trel. and its type locality to be in the Sierra de la Breña in northwestern Chihuahua.

En su revisión taxonómica de los encinos de América, Trelease (1924) propuso simultáneamente los nombres infraespecíficos Quercus oblongifolia Torr. var. pallidinervis en su circunscripción de la especie, y Q. oblongifolia f. pallidinervis en la clave para las especies en la serie Oblongifoliae (pp. 90, 91). El mencionado autor citó solamente dos colectas, ambas recogidas antes del presente siglo en el estado de Chihuahua. Tales muestras son las únicas que se han identificado como pertenecientes a esta variedad. Una colecta (Pringle 1571, Sept. 4, 1887, GH!, A!) procedente de la parte oeste-central de Chihuahua, de la montaña La Bufa, contigua al poblado de Cusihuiriachic, y otra (Nelson 6309, Ag. 25, 1899, GH!, US!) se realizó también en Chihuahua, pero la etiqueta de la muestra indica solamente la Sierra Madre como localidad de origen y no especifica datos más precisos. En la leyenda (p. 220) de la lámina número 146, Trelease indica que la colección de Nelson es el tipo y que ésta fue fotografiada en el herbario de "Washington" (US). Sin embargo, la ilustración correspondiente (Fig. 146) es exactamente igual a la muestra depositada en el Herbario Gray (GH); en este ejemplar la bellota se encuentra entre una hoja comida en parte por un insecto y una hoja doblada, en la ramita al lado derecho. Por consiguiente, la muestra de GH es el holotipo y el espécimen del herbario US corresponde al isotipo, puesto que claramente no es el representado en la Figura 146 de Trelease. Las anotaciones en ambos ejemplares son de la letra de Trelease. 
La localidad de la colección de Nelson se ubica en el noroeste de Chihuahua, aproximadamente $250 \mathrm{~km}$ en línea recta al noroeste del sitio de la colecta de Pringle. Goldman (1951, p. 123, 124) escribió que en la feçha 25 de agosto de 1899 la expedición acampó cerca de un aserradero operado por Mormones a 8000 pies $(2440 \mathrm{~m})$ de altitud en la Sierra de la Breña y al día siguiente se alejó de las montañas con rumbo a Nuevo Casas Grandes. Esta Sierra está situada cerca de $30^{\circ} 07^{\prime} \mathrm{N}, 108^{\circ} 10^{\prime} \mathrm{W}$, a la orilla oriental del cuerpo principal de la Sierra Madre Occidental (mapa de Chihuahua 08, 1:1,000,000, 1985, Instituto Nacional de Estadística, Geografía e Informática, corregido el error de grados de latitud), unos $45 \mathrm{~km}$ directamente al suroeste de Nuevo Casas Grandes. La altitud del campamento se encuentra por arriba del límite de la distribución conocida de $Q$. oblongifolia en esta latitud.

Las dos colectas mencionadas son muy semejantes a las muestras de Quercus depressipes Trel. que fueron recogidas en La Bufa, contigua a Cusihuiriachic (Spellenberg \& Zimmerman 8590 CAS, MEXU, NMC) y a unos pocos kilómetros al noroeste de la localidad del paratipo de Q. oblongifolia var. pallidinervis (Spellenberg et al. 9172, CAS, CIIDIR, MEXU, -NMC, NY; 9191 CIIDIR, MEXU, NMC).

En consecuencia esta variedad debe ser considerada como sinónimo del arbusto pequeño y rizomatoso, $Q$. depressipes. Las hojas de esta especie y de la variedad pallidinervis son obovadas, delgadas, dentadas, glaucas y casi glabras. Por otra parte Quercus oblongifolia, que es un árbol usualmente con hojas ovaladas, sin dientes y más coriáceas, no se encontró en ninguna de las dos áreas donde las colectas originales de la var. pallidinervis habían sido recogidas.

\section{AGRADECIMIENTOS}

El autor agradece a los Drs. Cecilio y Rosalinda Barrera y Jorge Nocedal por su ayuda en la traducción de inglés a español.

\section{LITERATURA CITADA}

Goldman, E. A. 1951. Biological investigations in Mexico. Smithsonian Misc. Coll. 115. 426 pp. Trelease, W. T. 1924. The American oaks. Mem. Nat. Acad. Sci. 20. 255 pp. + 420 ilustraciones. 